\title{
Calibrating Thrombin Generation in Different Samples: Less Effort with a Less Efficient Substrate
}

\author{
Hans Jürgen Tapp, Claudia Grundmann, Manuela Kusch and Herbert König*
}

Paul Ehrlich Institut, Paul Ehrlich Str. 51-59, D-63225 Langen, Germany

\begin{abstract}
The technique of thrombin generation has been established as a promising tool for the diagnosis, risk estimation, and perhaps prognosis of coagulation insufficiencies. Without further experimentation an indication can be obtained if a sample donor carries the risk for haemophilia or thrombophilia, either congenital or acquired. A comparison of two different thrombin generation assays is presented, demonstrating that a currently not commercially available test allows an easier and cheaper calibration than its commercial counterpart. This is achieved by employment of a less efficient but highly specific peptide substrate for thrombin and the involvement of low amounts of analyte (plasma samples).
\end{abstract}

\section{INTRODUCTION}

Clotting tests were almost the sole tests in the past for the diagnosis of coagulation disturbances and are still in use. These tests have their natural end point at a time when only $3-5 \%$ of thrombin has been formed. Although it was developed half a century ago $[1,2]$, it is only during the last one and a half decade that the thrombin generation test has attracted increasing attention. It appears that this test may replace coagulation based tests in many cases in the future $[3,4]$, more so as computer aided models of the events leading to coagulation have been developed and been shown to correlate well with biochemical observations $[5,6]$.

This expectation appears justified, as the thrombin generation test has since improved significantly by the introduction of continuous techniques employing chromogenic and fluorogenic substrates [7-10], thus replacing the original subsampling techniques which are laborious and time consuming. Simultaneous handling of many samples is possible by this method, thus saving time and costs. Fluorescence based applications have now the widest distribution because these techniques have advantages over the absorption based approaches as the signal in the latter is disturbed by turbidity caused by coagulation events. It is now possible to perform measurements for the characterization of hereditary or acquired coagulation disturbances as well as for investigations into the influence of various medicines that affect the coagulation process beyond the time of clotting [9-19]. Hemker et al. have reviewed further literature on such applications [20]. Thus, this test may turn out to be a valuable tool not only for the diagnosis of haemostatic disturbances but also for the prognosis of such conditions as hemophilia or thrombophilia. This test may as well become a tool for the surveillance of therapeutic regimes aimed at correcting such defects.

For the diagnosis and, if necessary, treatment of a coagulation defect comparable data from a normal coagulation process are required to serve as a reference. Therefore,

\footnotetext{
*Address correspondence to this author at the Paul Ehrlich Institut, Paul Ehrlich Str. 51-59, D-63225 Langen, Germany; Tel: +496103772720; E-mail:koehr@pei.de
}

exactly characterized normal plasmas should be available. The fact that different plasmas have different absorption characteristics when examined in a thrombin generation experiment complicates such a comparative characterization. Depending on the fluorometer used, the fluorescence signal may not be proportional to the fluorophore above a certain signal level (inner filter effect). Furthermore, it is difficult to discriminate whether the slope of the fluorescence signal observed during a thrombin generation experiment levels off due to thermal enzyme inactivation or inhibition of thrombin by plasma inhibitors such as alpha- 2 macroglobulin $(\alpha 2 \mathrm{M})$ or antithrombin (AT). Substrate exhaustion may also account for this effect. Our approach to measure thrombin generation differs essentially from similar approaches $(8,9$, Technothrombin ${ }^{\circledR}$ TGA, respectively), in that we use a much less efficient substrate and employ significantly lower plasma concentrations during the assay $[10,21]$. We have compared these different thrombin generation assay types employing simple model systems aimed at identifying the contribution of the aforementioned limitations to the overall performance. Consequently, it was possible to identify the pros and cons of the two different approaches. We now report that our approach to use an inefficient (slowly cleaved) but highly specific substrate (recognized by thrombin, but much less by other proteases) together with a reduced volume of plasma results in a very simple algorithm to calibrate the obtained thrombin generation data.

\section{MATERIALS AND METHODS}

\section{Materials}

Blood was obtained from healthy individuals as well as donors with known coagulation defects from our institute. Nine volumes blood were drawn with minimal suction into one volume $106 \mathrm{mM}$ trisodium citrate (Sarstedt, Nuembrecht, Germany). Vacuum containers were avoided as plasma prepared by this method often showed altered thrombin generation behaviour, which may be due to activation events during this form of blood collection. Platelet poor plasma was prepared from these blood donations by two consecutive centrifugations (200x g for 20 minutes, followed by $2400 \mathrm{xg}$ for 20 minutes at room temperature) within 60 minutes after venepuncture. The upper half of the resultant 
supernatant was collected each time. Commercially available plasma samples served as "normal plasma" (NP).

The fluorogenic substrates Boc- Val-Arg- AMC (B-VRAMC), Z- Gly-Gly-Arg- AMC (Z-GGR-AMC), and their split product 7- amino- 4- methylcoumarin (AMC) were obtained from Bachem (Weil am Rhein, Germany). Both peptide substrates were originally developed and characterized by Kawabata et al. [22]. In this work, they compared a panel of 74 substrates with respect to their conversion by several serine proteases.

Phospholipids with tissue factor ("Innovin") were from Dade Behring (Marburg, Germany). Phospholipids without tissue factor ("Cephalin") were from Boehringer- Roche, (Mannheim, Germany). These were mixed to give a final tissue factor concentration of $2 \mathrm{pM}$.

Thrombin was obtained from Merck/ Calbiochem (Schwalbach, Germany) and was active site titrated with 4methylumbeliferyl- p- guanidinobenzoate (Sigma, Deisenhofen, Germany) essentially as described [23]. Human serum albumin ("Human albumin 20\% Behring", hSA) was from Aventis/ Behring (Marburg, Germany). All other chemicals were obtained in p.A. - grade from different manufacturers.

Fluorescence measurements were performed on a Fluoroscan Ascent (Thermo electron, Dreieich, Germany). Readings were taken at 360nm excitation, $440 \mathrm{~nm}$ emission. Selected experiments were analyzed in three further fluorescence photometers (Safire, Infinite F 200, both from Tecan (Crailsheim, Germany), and an extra Fluoroscan Ascent). These were also used at excitation wavelength near $360 \mathrm{~nm}$ and emission wavelength near $440 \mathrm{~nm}$. Chromogenic reactions were monitored on a thermomax photometer (Molecular devices, Ismaning, Munich, Germany).

\section{METHODS}

\section{Thrombin Generation Assay}

The thrombin generation assay with B-VR-AMC as substrate was carried out essentially as described earlier [10, 21]. Tissue factor was reduced to $2 \mathrm{pM}$ final concentration. The assay volume in these experiments was $100 \mu 1$, containing $25 \mu \mathrm{l}$ plasma. For comparison, Z-GGR-AMC replaced BVR-AMC in some experiments. In these instances the assay volume $(120 \mu \mathrm{l})$ as well as the plasma volume $(80 \mu \mathrm{l})$ was adapted to the conditions described by Hemker et al. [20]. The buffer used was Hepes (2-[4-(2-Hydroxyethyl)-1piperazinyl]- ethanesulfonic acid) buffered saline $\mathrm{pH} 7,4$ containing 1\% hSA (HBS-hSA). All tests were conducted in triplicate. For reproducibility determinations we performed thrombin generation assays on three different days, each in triplicate. The data were normalized to normal plasma in order to compensate for e.g. temperature fluctuations between experiments.

\section{Kinetics of Thrombin}

Purified, active site titrated thrombin (from $44 \mathrm{nM}$ to 350 $\mathrm{nM})$ was measured over $1 \mathrm{~h}$ at $37^{\circ} \mathrm{C}$ in HBS-hSA $(100 \mu \mathrm{l}$ final volume) with B-VR-AMC and Z-GGR-AMC as substrate $(460 \mu \mathrm{M})$. Measurements were taken every $20 \mathrm{sec}-$ onds. For the determination of $\mathrm{K}_{\mathrm{M}}$ we varied the substrate concentrations from 0 to $1 \mathrm{mM}$ while the thrombin concentration was kept constant at $25 \mathrm{nM}$. The resultant velocity curves were evaluated using the GraphPad Prism software version 4.0. For the determination of $\mathrm{k}_{\mathrm{cat}}$ we determined the liberation of AMC from the respective substrates at 10 fold $\mathrm{K}_{\mathrm{M}}$ and thrombin at $25 \mathrm{nM}$.

\section{Dose Response Relationship}

Fluorescence based experiments are eventually hampered by the inner filter effect or quenching, which means that the fluorescence/ fluorophore relationship is not linear above a certain level characteristic for a given experimental setup [24]. To find out whether this phenomenon is at least partially accountable for the fading slope during a thrombin generation experiment, we determined the fluorescence raised by increasing amounts of AMC. The concentration of AMC diluted with HBS-hSA was varied to yield 0- 700 FU in that buffer. In the same way we determined the fluorescence obtained by these AMC-concentrations in different plasmas matching the conditions of our approach for thrombin generation experiments.

\section{Influence of the Fluorogenic Substrates on AT- Mediated Inhibition of Thrombin}

Thrombin $(5 \mathrm{nM})$ was incubated with and without AT $(13 \mathrm{nM})$ in presence and absence of the respective fluorogenic substrates $(460 \mu \mathrm{M})$. After one hour at $37^{\circ} \mathrm{C}$ the chromogenic substrate for thrombin S2238 was added $(1 \mathrm{mM}$, $\left.\mathrm{K}_{\mathrm{M}}=7 \mu \mathrm{M}, \mathrm{k}_{\mathrm{cat}}=170\right)$ and the residual activity of thrombin was determined over 10 minutes at $405 \mathrm{~nm}$.

\section{RESULTS}

\section{Thrombin Generation Curves}

In a first approach for the comparison of the two different thrombin generation assays, we have applied the two techniques to commercially available reference plasma, three different plasmas from normal donors and one from a person with thromboembolic history. Thrombin generation measurements were performed according to Hanker- Dusel et al. [10] and Hemker et al. [20], respectively. The data are presented as obtained without any attempt to correct for substrate exhaustion or inner filter effects as suggested by Hemker et al. [20]. We determined the coefficient of variation for peak thrombin in our thrombin generation test to be $1.2-2 \%$ (intra- assay) and 2- $5 \%$ (inter- assay). This value compares well to the one obtained by Gerotziafas et al. [25]. Both techniques yield similar if not identical time to peak with the corresponding plasmas but the obtained curves differ with respect to the much higher slope (approximately tenfold) obtained with the more efficient substrate Z-GGR-AMC (Fig. 1, bottom) as compared to B-VR-AMC (Fig. 1, top). Note the different scaling on the ordinates. It is also obvious that the derivatives of both curves differ in the steepness of the descending part of the curves. Both aspects, namely higher signal with Z-GGR-AMC, as well as the faster decay observed with this substrate were addressed in the subsequent experiments.

\section{Kinetics of Thrombin}

We considered it probable that a comparison of the two substrates with isolated thrombin would help in characterization of the differences observed in the thrombin generation experiment. For this purpose we incubated various thrombin 


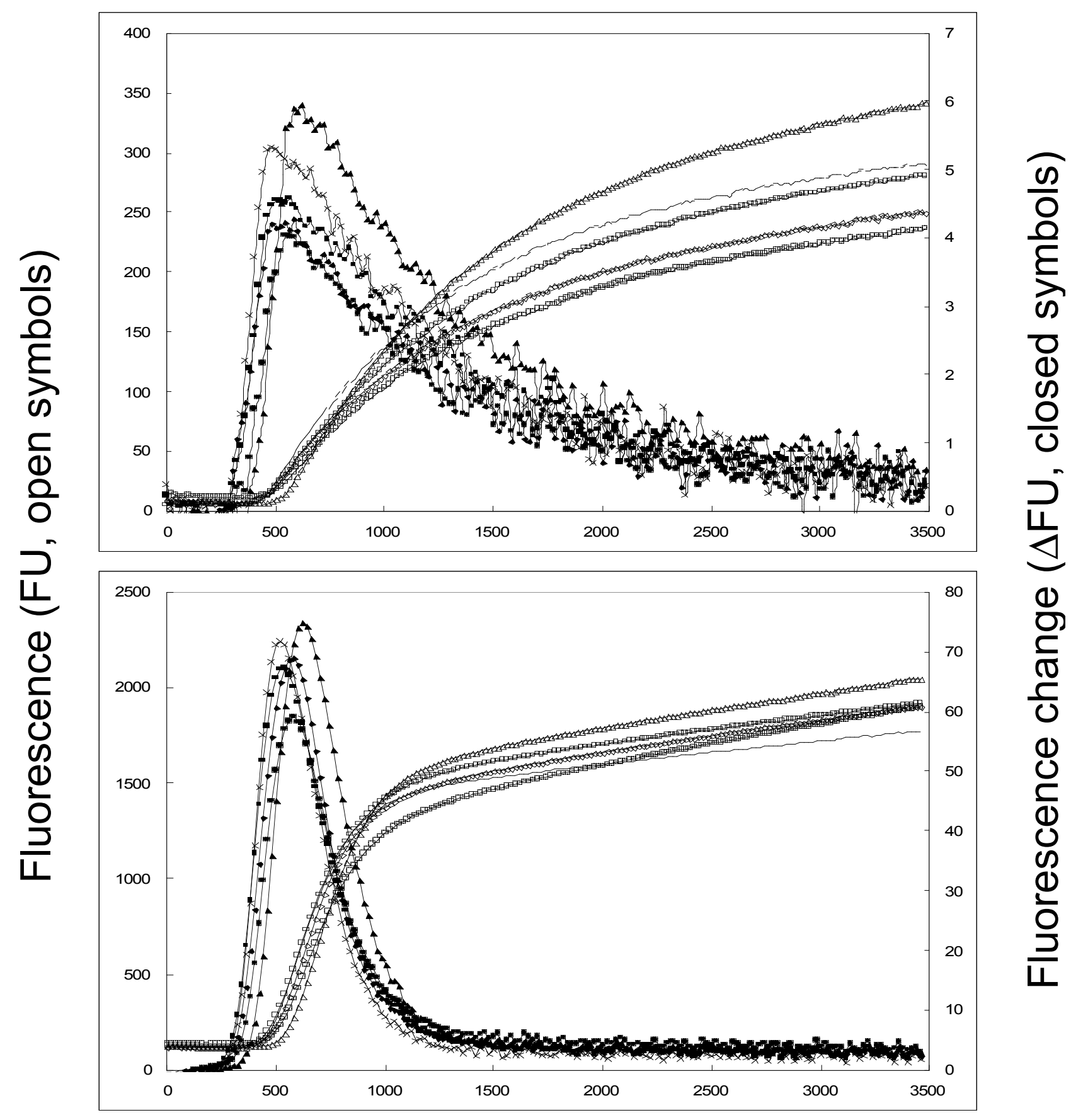

\section{Time (seconds)}

Fig. (1). Typical thrombin generation results. Thrombin generation experiments were done according to [10, top] and [20, bottom]. The major differences between these assay types are that different plasma volumes and different substrates are used (Boc-Val-Arg-AMC and 25 $\mu 1$ plasma according to [10], or Z-Gly-Gly-Arg-AMC and $80 \mu \mathrm{l}$ plasma according to [20]). One plasma pool (Diamonds) and four individual plasma samples (squares, triangles, circles, plain line/ crosses) were assayed by each method. Among the individual samples is one with proven thrombophilia (triangles). The thrombin generation curves as measured (left y-axis) and their respective first derivatives (right yaxis) are shown in an overlay plot for both assay types. Identical symbols are used for the different fluorescence traces and their respective derivatives (open symbols: FU, closed symbols: $\Delta \mathrm{FU}$ ). The abscissa represents the time (calibrated in seconds).

concentrations with the substrates in question. Both substrates were present at $460 \mu \mathrm{M}$ and the reaction was allowed to proceed for 60 minutes. As shown in Fig. (2) the more efficient substrate Z-GGR-AMC (triangles) yields saturating curves at all thrombin concentrations tested, while with BVR-AMC (circles) as the substrate, no saturation is reached 


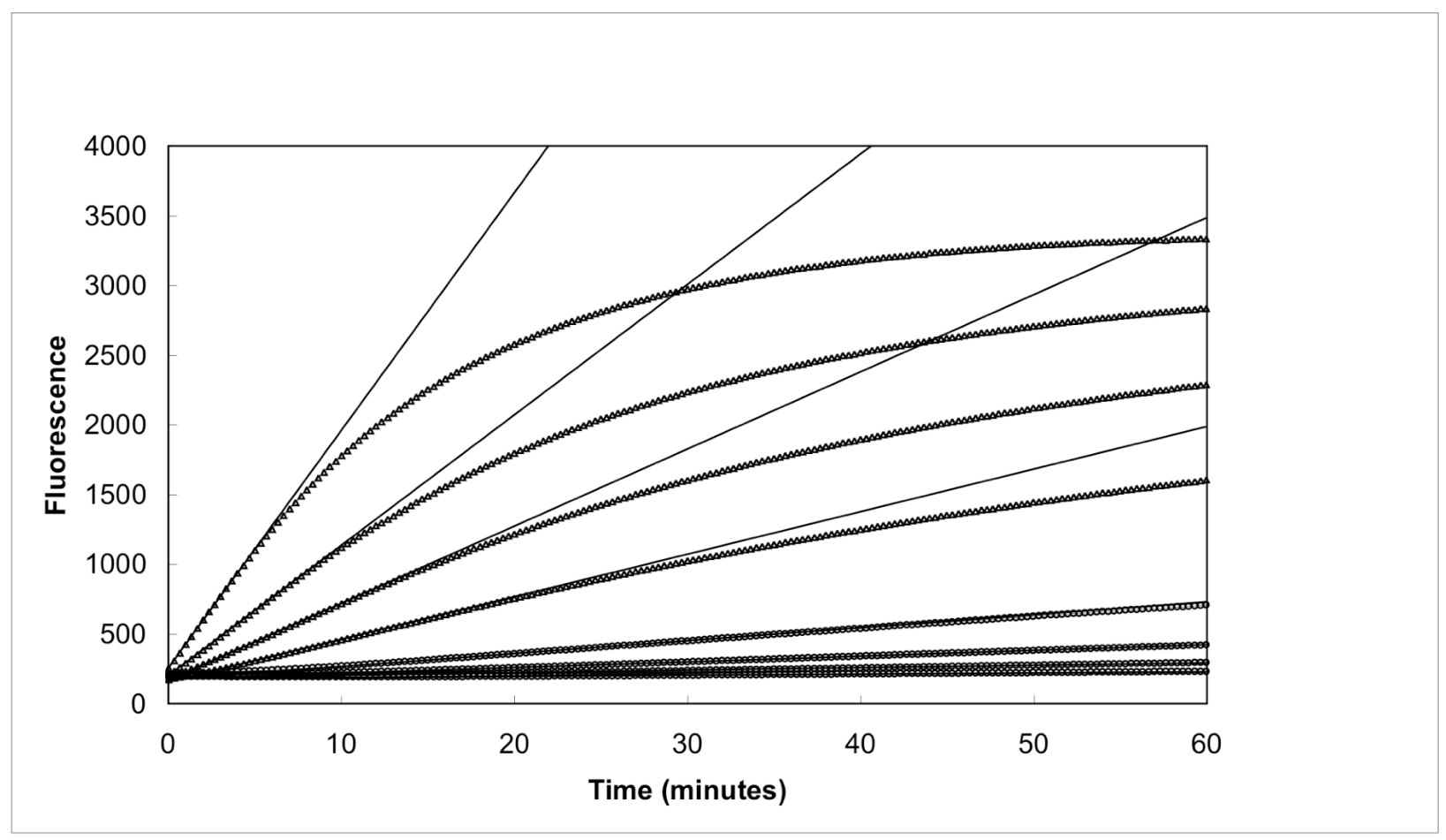

Fig. (2). Kinetics of purified thrombin with two different substrates. Purified thrombin (44- $350 \mathrm{nM}$ ) was allowed to cleave Z-Gly-Gly-ArgAMC (triangles) or Boc-Val-Arg-AMC (circles) for one hour at $37^{\circ} \mathrm{C}$ in HBS-hSA. Both substrates were present at an initial concentration of $460 \mathrm{nM}$. The tangents (initial velocity) to the respective curves are drawn as thin lines.

during the whole time interval investigated. The tangents (plain lines) to the respective curves during the initial few minutes (initial velocity) were included to aid the comparison.

As substrate turnover is influenced by $\mathrm{K}_{\mathrm{M}}$ and $\mathrm{k}_{\text {cat }}$, we determined both parameters for thrombin with both substrates. With Z-GGR-AMC we found $\mathrm{K}_{\mathrm{M}}=100 \mu \mathrm{M}$, $\mathrm{k}_{\text {cat }}=$ 1.03 , with B-VR-AMC as substrate the respective values were $160 \mu \mathrm{M}$ and 0.032 .

While the $\mathrm{K}_{\mathrm{M}}$ of both substrates are of the same order and are three- to fourfold lower than the actual substrate concentration, we consider it unlikely that this difference causes the protracted curve with B-VR-AMC. The much more pronounced difference between the respective $\mathrm{k}_{\mathrm{cat}}$ could be responsible for this effect. We therefore measured the inhibitory effect of both substrates on the AT mediated inhibition of thrombin.

Both substrates interfere similarly with AT inhibition of thrombin (residual activity without substrate: $49 \%$, with ZGGR-AMC: $79.9 \%$, with B-VR-AMC: $82.6 \%$ ). These values are corrected for the (negligible) effect of the fluorogenic substrates on the cleavage of the chromogenic substrate S2238. While this work was in progress, we learned that dilution of plasma results in diminishing the inhibitory potential of tissue factor pathway inhibitor [26].

\section{Determination of the Quench Effect Brought About by Plasma}

The results illustrated in Fig. (2) are compatible with the idea that in the thrombin generation experiment involving ZGGR-AMC the substrate becomes limiting whereas in the experiment with B-VR-AMC it does not. Another possibil- ity, that thrombin is not stable during the investigated period of time at the chosen conditions $\left(37^{\circ} \mathrm{C}\right)$, is disproved by the fact that in the presence of B-VR-AMC, thrombin maintains its activity over the whole time interval followed. A third explanation would be that the phenomenon of the inner filter effect causes the discrepancy between the two substrates. We investigated the effect of different plasmas on fluorescence recovery in a spiking experiment similar to a thrombin generation assay. In this experiment AMC, the split product of the fluorogenic substrates, replaced the trigger for thrombin generation (tissue factor). In addition to buffer, which served as the reference, we used several plasmas (the same as those investigated in the experiment for Fig. (1)) for fluorescence measurements after spiking the samples with $\mathrm{AMC}$ of known concentration. We calculated the AMC range to elicit a fluorescence signal similar to the one observed in a typical thrombin generation experiment with B-VR-AMC ( $\sim 350 \mathrm{FU}$ in plasma or $\sim 700 \mathrm{FU}$ in buffer). In all the plasmas tested, the fluorescence response is linear over the whole range tested. On the other hand, the recovery differs depending on the plasma investigated, indicating different quench effects caused by these plasmas (Fig. 3, Table 1). In independent experiments (not shown) we found that the most prominent plasma protein, albumin, plays a major role in this respect. Moreover, higher plasma concentrations (such as those utilized by Hemker et al. [20]) yielded even less fluorescence. Another observation is noteworthy (not shown), namely that essentially the same results are obtained when coagulation is allowed to occur. In these experiments AMC replaced BVR-AMC, while tissue factor was added to the reaction (besides Calcium). Only a slight and short disturbance of the signal was observed at a time when coagulation was expected. We have reported a similar observation already [10]. 


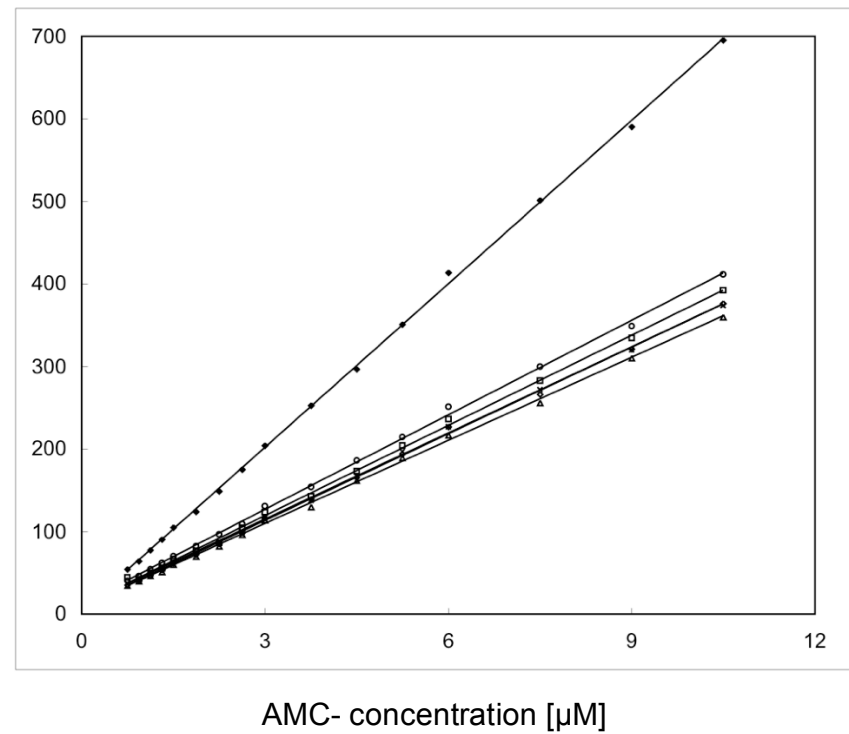

Fig. (3). Quenching of fluorescence caused by individual plasmas. Plasma samples (same as those used in Fig. (1)) were mixed with varying amounts of AMC (the split product of Z-Gly-Gly-ArgAMC or Boc-Val-Arg-AMC) to yield the final concentrations of $\mathrm{AMC}$ as indicated on the $\mathrm{x}$ - axis. HBS-hSA served as reference (closed diamonds). Although no significant change of the fluorescent signal was expected, we treated this experiment as a kinetic one, in order to check for deviations of the signal.

Table 1. Calculation of the Actual Thrombin Concentration

\begin{tabular}{|c|c|c|c|}
\hline & Mean Recovery (\%) & Std Dev & Correction Factor \\
\hline \hline NP & 57 & 0,03 & 1,74 \\
\hline a & 61 & 0,06 & 1,64 \\
\hline b & 56 & 0,03 & 1,79 \\
\hline c & 65 & 0,05 & 1,55 \\
\hline d & 59 & 0,04 & 1,70 \\
\hline
\end{tabular}

Based on the results presented in Fig. (3) the mean recovery of fluorescence in individual plasmas is calculated (given in \%). By multiplying the values obtained in the thrombin generation experiment with the correction factor ( $=100 /$ mean recovery), the unquenched values are obtained. Data are rounded to two decimal places.

\section{DISCUSSION}

We originally developed the fluorescent thrombin generation assay as an approach for the evaluation of thrombogenicity associated with certain coagulation factor concentrates e.g. prothrombin complex concentrates $[10,21]$. We took advantage of the work of Kawabata et al. [22], who have characterized a large panel of AMC- peptides with respect to their use by several serine proteases. Because the physiological thrombin generation should ideally not be influenced at all (in practice: minimally), we chose the substrate with highest specificity and a low affinity as possible for thrombin. Among the substrates tested in that publication, B-VR-AMC best fulfilled this demand. The fact that this substrate yields low fluorescence levels during the course of a thrombin generation experiment may appear at first disadvantageous. We have nevertheless chosen this substrate, originally because of its unsurpassed selectivity for thrombin when compared to other substrates in the above mentioned publication. In particular, the comparison with ZGGR-AMC proves this substrate to be the better choice. In our previous publications we concentrated on thrombogenicity in certain coagulation factor concentrates and therefore neglected the problem of inner filter effect, as our investigations were performed almost exclusively using a model plasma as the matrix and variations were introduced by the addition of trace amounts of factor concentrates. Since we plan to expand our investigations into individual plasma samples, it appeared justified to examine whether our test system requires modifications as described by Hemker et al. [20].

While we were less concerned about problems with substrate exhaustion in our assay (one of the benefits of a poor substrate), we considered it necessary to examine if different plasma samples have different influence on fluorescence recovery (inner filter effect, quenching). Our experiments show that substrate exhaustion in fact did not occur with our approach (Fig. 2), while with Z-GGR-AMC this effect has already been described [20]. The succeeding experiments then addressed the question: Does the inner filter effect lead to an underestimation of enzyme activity with increasing fluorescence and/ or increasing amounts of plasma? While the Hemker group in addition to substrate exhaustion apparently observed this, this was evidently not the case with our approach. This is obvious from Figs. $(\mathbf{2}, \mathbf{3})$, where we show that the fluorescence increases linearly with the fluorophore in buffer and linearly in plasma over the range tested, i.e. that range obtained in a thrombin generation experiment performed according to our procedure. Additional experiments $\left(\mathrm{K}_{\mathrm{M}^{-}}\right.$and $\mathrm{k}_{\mathrm{cat}}{ }^{-}$determinations as well as inhibition of AT mediated thrombin inhibition by the substrates do not support the assumption that B-VR-AMC competes significantly more efficient with physiological reactions than ZGGR-AMC. We do show, however, that both substrates interfere with the physiological reactions and thus confirm the notion of Butenas and Mann [27].

How can our results be utilized in order to determine correct thrombin levels during the course of a thrombin generation experiment? The answer to this question is given in Table 1, where we show that

i) Each plasma has a certain and characteristic quench effect on fluorescence and

ii) This effect can be expressed as a constant value which is easily converted into a correction factor that may be used for recalculation of thrombin concentration. Thus, one can correct any individual plasma sample for its quench effect and correlate it to normal plasma (a pool of many donors) that is defined as $100 \%$. This proceeding would also take into account the suggestion of Butenas and Mann [27]. We conclude that others may easily adopt our approach to measure thrombin generation without the necessity to prepare or buy a special calibrator such as the thrombin- $\alpha 2$ macroglobulin complex. Besides, no special software is needed to compensate for insufficiencies caused by the use of suboptimal substrate and unnecessary large sample volume. We also found that our approach is not limited to the use of a specific 
fluorometer, but may be performed using equipment of other vendors.

\section{ACKNOWLEDGEMENT}

We thank Prof. R. Seitz for encouragement and continuous support.

\section{REFERENCES}

[1] Macfarlane RG, Biggs R. A thrombin generation test. J Clin Pathol 1953; 6: 3-7

[2] Pitney WR, Dacie J. A simple method of studying the generation of thrombin in recalcified plasma. J Clin Pathol 1953; 6: 9-13.

[3] Hemker HC, Wielders S, Kessels H, Beguin S. Continuous registration of thrombin generation in plasma, its use for the determination of the thrombin potential. Thromb Haemost 1993; 70: 617-24.

[4] Wielders S, Mukherjee M, Michiels J, et al. The routine determination of the endogenous thrombin potential, first results in different forms of hyper- and hypocoagulability. Thromb Haemost 1997; 77(4): 629-36.

[5] Van't Veer C, Mann KG. Regulation of tissue factor initiated thrombin generation by the stoichiometric inhibitors tissue factor pathway inhibitor, antithrombin III, and heparin cofactor II. J Biol Chem 1997; 272(7): 4367-77.

[6] Hockin MF, Jones KC, Everse SJ, Mann KG. A model fort he stoichiometric regulation of blood coagulation. J Biol Chem 2002; 277(21): 18322-33.

[7] Ramjee MK. The use of fluorogenic substrates to monitor thrombin generation for the analysis of plasma and whole blood coagulation. Anal Biochem 2000; 277: 11-8.

[8] Hemker HC, Giesen OL, Ramjee M, Wagenvoord R, Beguin S. The thrombogram: monitoring thrombin generation in platelet rich plasma. Thromb Haemost 2000; 83: 589-91.

[9] Varadi K, Negrier C, Berntorp E, et al. Monitoring the bioavailability of FEIBA with a thrombin generation assay. J Thromb Haemost 2003; 1: 2374-80.

[10] Hanker DC, Grundmann C, Eich S, Seitz R, König H. Identification of prothrombin as major thrombogenic agent in prothrombin complex concentrates. Blood Coagul Fibrinolysis 2004; 15(5): 40511.

[11] Al Dieri R, Peyvandi F, Santagostino E, et al. The thrombogram in rare inherited coagulation disorders: its relation to clinical bleeding. Thromb Haemost 2002; 88: 576-82.

[12] Keularts IMLW, Zivelin A, Seligson U, Hemker HC, Beguin S. The role of factor XI in thrombin generation induced by low concentrations of tissue factor. Thromb Haemost 2001; 85: 1060-5.

[13] Kyrle PA, Mannhalter C, Beguin S, et al. Clinical studies and thrombin generation in patients homozygous or heterozygous for the G20210A mutation in the prothrombin gene. Arterioscler Thromb Vasc Biol 1998; 18: 1287-91.
[14] Sarich TC, Eriksson UG, Mattsson C, et al. Inhibition of thrombin generation by the oral direct thrombin inhibitor ximelagatran in shed blood from healthy male subjects. Thromb Haemost 2002; 87: 300-5.

[15] Rosing J, Tans G, Nicolaes GA, et al. Oral contraceptives and venous thrombosis: different sensitivities to activated protein $\mathrm{C}$ in women using second- and third- generation oral contraceptives. $\mathrm{Br}$ J Haematol 1997; 97: 233-8.

[16] Beltran- Miranda CP, Khan A, Jaloma- Cruz AR, Laffan MA Thrombin generation and phenotypic correlation in hemophilia A. Hemophilia 2005; 11: 326-34.

[17] Tchaikovski SN, van Vliet HAAM, Thomassen CLGD, et al. Effect of oral contraceptives on thrombin generation measured via calibrated automated thrombography. Thromb Haemost 2007; 98 : 1350-6.

[18] Dargaud Y, Trzeciak MC, Bordet JC, Ninet J, Negrier C. Use of calibrated automated thrombinography $+/$ - thrombomodulin to recognize the prothrombotic phenotype. Thromb Haemost 2006; 96: 562-7.

[19] Tripodi A, Martinelli I, Chantarangkul V, Battaglioli T, Clerici M, Mannucci PM. The endogenous thrombin potential and the risk of venous thromboembolism. Thromb Res 2007; 121: 353-9.

[20] Hemker HC, Giesen P, Al Dieri R, et al. Calibrated automated thrombin generation measurement in clotting plasma. Pathophysiol Haemost Thromb 2003; 33: 4-15.

[21] Grundmann C, Plesker R, Kusch M, et al. Prothrombin overload causes thromboembolic complications in prothrombin complex concentrates: In vitro and in vivo evidence. Thromb Haemost 2005; 94: 1338-9.

[22] Kawabata S, Miura T, Morita T, et al. Highly sensitive peptide-4methylcoumaryl 7 -amide substrates for blood clotting proteases and trypsin. Eur J Biochem 1988; 172: 17-25.

[23] Chase T, Shaw E. Titration of trypsin, plasmin, and thrombin with p- nitrophenyl p'-guanidinobenzoate $\mathrm{HCl}$. In: Perlmann PE, Lorand L, Eds. Methods enzymology. New York: Academic Press 1970; 19: pp. 20-7.

[24] Wehry EL. Molecular fluorescence and phosphorescence spectrometry. In: Settle F, Ed. Handbook of instrumental techniques for analytical chemistry. New Jersey: Prentice- Hall, Inc. A Simon and Schuster Company Upper Saddle River 1997; pp. 507-39.

[25] Gerotziafas GT, Depasse F, Busson J, Leflem L, Elalamy I, Samama MM. Towards a standardization of thrombin generation assessment: the influence of tissue factor, platelets and phospholipids concentration on the normal values of Thrombogram- Thrombinoscope assay. Thromb J 2005; 3: 16.

[26] De Smedt E, Wagenvoord R, Hemker HC. The technique of measuring thrombin generation with fluorogenic substrates: 3 . The effect of sample dilution. Thromb Haemost 2009; 101: 165-70.

[27] Butenas S, Mann KG. Caution in the interpretation of continuous thrombin generation assays. J Thromb Haemost 2007; 5: 1084-5. 